Comparing Risks by Acceptance and Rejection

Author(s): Sergiu Hart

Source: Journal of Political Economy, Vol. 119, No. 4 (August 2011), pp. 617-638

Published by: The University of Chicago Press

Stable URL: http://www.jstor.org/stable/10.1086/662222

Accessed: 15/10/2011 13:17

Your use of the JSTOR archive indicates your acceptance of the Terms \& Conditions of Use, available at http://www.jstor.org/page/info/about/policies/terms.jsp

JSTOR is a not-for-profit service that helps scholars, researchers, and students discover, use, and build upon a wide range of content in a trusted digital archive. We use information technology and tools to increase productivity and facilitate new forms of scholarship. For more information about JSTOR, please contact support@jstor.org. 


\title{
Comparing Risks by Acceptance and Rejection
}

\author{
Sergiu Hart
}

Hebrew University of Jerusalem

\begin{abstract}
Stochastic dominance is a partial order on risky assets ("gambles") that is based on the uniform preference-of all decision-makers in an appropriate class-for one gamble over another. We modify this requirement, first, by taking into account the status quo (given by the current wealth) and the possibility of rejecting gambles, and second, by comparing rejections that are substantive (i.e., uniform over wealth levels or over utilities). This yields two new stochastic orders: "wealthuniform dominance" and "utility-uniform dominance." Unlike stochastic dominance, these two orders are complete: any two gambles can be compared. Moreover, they are equivalent to the orders induced by, respectively, the Aumann-Serrano index of riskiness and the Foster-Hart measure of riskiness.
\end{abstract}

\section{Introduction}

A risky asset (or gamble) yields uncertain returns according to a given probability distribution; these returns may be positive (gains) or negative (losses). ${ }^{1}$ How can gambles be compared to one another? Which is "less risky"? While different decision-makers regard gambles differentlyeach according to his own risk posture-we would like to capture in

Previous versions: December 2009 (Discussion Paper no. 531, Center for the Study of Rationality), December 2010. This paper supersedes "A Simple Riskiness Order Leading to the Aumann-Serrano Index of Riskiness," May 2008 (draft), August 2009 (Discussion Paper no. 517, Center for the Study of Rationality). Research was partially supported by grants of the Israel Science Foundation and the European Research Council. The author thanks Bob Aumann, Elchanan Ben-Porath, Dean Foster, Werner Hildenbrand, Gil Kalai, Haim Levy, Moti Michaeli, Phil Reny, Frank Riedel, Yossi Rinott, Benjy Weiss, Shlomo Yitzhaki, and the anonymous referees, for useful discussions and suggestions.

1 The outcomes of a gamble should be understood as net changes to the current wealth, and not as the final wealth. 
these comparisons the gambles' inherent riskiness. That is, we want to find objective ways to compare gambles: independently of the specific decision-maker, and depending only on the gambles themselves (i.e., on their outcomes and probabilities). Such objective comparisons do exist, for instance, for the "return" of gambles (compare their expectations) and their "spread" (compare their variances). Moreover, these comparisons yield complete orders (i.e., any two gambles can be compared). The aim of this paper is to do the same for riskiness-that is, to provide complete and objective orders of riskiness.

Let $g$ and $h$ denote two gambles. There are cases where it is clear that $g$ is less risky than $h$; this is certainly so when $g$ is obtained from $h$ by increasing some gain, by decreasing some loss, or by replacing a lottery with its expectation. Combining these kinds of transformations yields the well-known second-degree stochastic dominance order (see Hadar and Russell 1969; Hanoch and Levy 1969; Rothschild and Stiglitz 1970, 1971; Machina and Rothschild 2008). As it turns out, there is an equivalent approach that leads to the same comparison: $g$ (second-degree) stochastically dominates $h$ if and only if all risk-averse decision-makers prefer ${ }^{2}$ $g$ to $h$. This is a natural approach since it is risk, after all, to which riskaverse decision-makers are averse, and this aversion can be used to compare the riskiness of the two gambles. However, it seldom happens that all decision-makers agree which one of the two gambles is preferred to the other. Indeed, in general some will prefer the first to the second, others the second to the first-and stochastic dominance is then silent. Formally, this means that the stochastic dominance order between gambles is a partial (rather than complete) order-in fact, very partial and far from complete.

Suppose now that decision-makers may choose whether to accept or reject a gamble. ${ }^{3}$ But then, if both $g$ and $h$ are rejected, does it really matter in this case that, say, $g$ is preferred to $h$ ? This suggests a way to weaken the requirement of stochastic dominance, by asking only that $g$ be accepted more, and thus rejected less, than $h$. We will say that $g$ acceptance dominates $h$ if every time that $g$ is rejected (by a risk-averse decision-maker) then so is $h$.

Clearly, the acceptance dominance requirement is a weakening of the stochastic dominance requirement: if $g$ is preferred to $h$ and $g$ is rejected, then surely $h$ is rejected (since the status quo is preferred to $g$, which

\footnotetext{
${ }^{2}$ To streamline the text, we say "greater than" rather than "greater than or equal to": all comparisons should thus be understood in the weak sense. For example, "prefers" means "prefers or is indifferent to," and "rejected less" means "rejected less often or as often." Also, all the orders we consider are weak orders (from which the strict part and the indifference part are easily deduced as usual).

${ }^{3}$ A gamble is rejected whenever staying put at the current wealth (the "status quo") is preferable to taking the gamble, and is accepted otherwise. See Sec. IV(c) for further discussion.
} 
in turn is preferred to $h$ ). Therefore the acceptance dominance order extends the stochastic dominance order. That it is an actual extension can be seen, for example, by taking a gamble $g$ with positive expectation and doubling its outcomes (i.e., multiplying all gains and losses by 2); the resulting gamble $h=2 g$ turns out always to be acceptance dominated by $g$, even though it can never be stochastically dominated by $g$ (see Sec. IV $(\mathrm{j})$ ).

While acceptance dominance allows one to compare more pairs of gambles than does stochastic dominance, it is still a very partial order: for general gambles $g$ and $h$, there are instances where $g$ is rejected and $h$ is accepted, and other instances where $h$ is rejected and $g$ is accepted. The reason is that the requirement that "in every instance that $g$ is rejected $h$ must also be rejected" is a strong requirement, and thus hard to satisfy for general gambles $g$ and $h$. After all, $g$ and $h$ may take very different values, with different ranges, and it may be too much to expect that for all utility functions and all wealth levels, the rejection of one gamble $g$ will always imply the rejection of another gamble $h$.

All this leads to the following idea: take into account rejection only when it is "substantive," in the sense that it occurs in a significant range of decision problems. That is, only a stronger, "uniform" rejection-not just a single instance, but rather a whole range of rejections-should qualify as evidence of the riskiness of gambles: the gamble that is uniformly rejected less is less risky and dominates the other. Since an acceptance or rejection decision is characterized by a utility function and a wealth level, there are two simple ways to impose uniformity: one in which the rejection is wealth-uniform (i.e., rejection by one utility function at all wealth levels), and the other in which it is utility-uniform (i.e., rejection at one wealth level by all utilities). ${ }^{4}$

We thus obtain two new orders on gambles, which we call "wealthuniform dominance" and "utility-uniform dominance." Formally, $g$ wealth-uniformly dominates $h$ if any risk-averse utility function that rejects $g$ at all wealth levels also rejects $h$ at all wealth levels; and $g$ utility-uniformly dominates $h$ if any wealth level at which all risk-averse utility functions reject $g$ is also a wealth level at which they all reject $h$. That is, in the former case $g$ is wealth-uniformly rejected less than $h$, and in the latter $g$ is utility-uniformly rejected less than $h$. Clearly, these two new orders extend acceptance dominance and thus, a fortiori, stochastic dominance: if every time that $g$ is rejected $h$ is also rejected, then any uniform rejection of $g$ implies the same uniform rejection of $h$.

What may come as a surprise is that each one of these two uniform

\footnotetext{
${ }^{4}$ To ensure the soundness of these uniform requirements some standard regularity conditions will be imposed on the class of utility functions that are considered; see Sec. II.B and the discussion in Sec. IV(d) and (e).
} 
dominance orders is in fact a complete order: any two gambles can be compared. That is, for any $g$ and $h$, either $g$ wealth-uniformly dominates $h$, or $h$ wealth-uniformly dominates $g$; also, either $g$ utility-uniformly dominates $h$, or $h$ utility-uniformly dominates $g$ (but in general the two orders may yield different comparisons). Thus, when one considers strong aversion to gambles (i.e., aversion that is uniform with respect to wealth or utility), there is no longer any ambiguity: of any two gambles, one is always rejected more.

Recently, two numerical measures of riskiness were introduced: the "economic" index $R^{\mathrm{AS}}$ developed by Aumann and Serrano (2008), and the "operational" measure $R^{\mathrm{FH}}$ of Foster and Hart (2009). We will show a noteworthy connection between our orders and these measures of riskiness: wealth-uniform dominance is equivalent to $R^{\mathrm{AS}}$, and utilitydominance to $R^{\mathrm{FH}}$. That is, $g$ wealth-uniformly dominates $h$ if and only if the AS-riskiness index $R^{\mathrm{AS}}(g)$ of $g$ is less than or equal to the ASriskiness index $R^{\mathrm{AS}}(h)$ of $h$, i.e., $R^{\mathrm{AS}}(g) \leq R^{\mathrm{AS}}(h)$; and $g$ utility-uniformly dominates $h$ if and only if the FH-riskiness measure $R^{\mathrm{FH}}(g)$ of $g$ is less than or equal to the FH-riskiness measure $R^{\mathrm{FH}}(h)$ of $h$, i.e., $R^{\mathrm{FH}}(g) \leq$ $R^{\mathrm{FH}}(h)$.

One can draw a parallel between the approaches to riskiness and standard decision and consumer theory. In the latter, there are two ways to model rational choice: one is based on comparing alternatives, which yields a "preference order"; the other is based on assigning a number to each alternative, which yields a "utility function." The connection between the two is that a utility function represents a preference order if and only if the preferred outcome has a higher utility. Similarly, in the context of riskiness, the present paper provides the "order" approach, while the papers of Aumann and Serrano (2008) and Foster and Hart (2009) provide the "numerical index" approach; the results stated in the previous paragraph yield the connection (see also the discussion in Sec. IV(a)).

Another interesting observation is that the two ways of getting uniform dominance further emphasize the "duality" between the AS-index and the FH-measure (pointed out in Foster and Hart 2009, Sec. VI.A, (iii)): the AS-index looks for the critical utility regardless of wealth, whereas the FH-measure looks for the critical wealth regardless of utility.

To summarize: taking into consideration the status quo given by the current wealth level and the possibility to reject gambles has enabled us to go beyond the standard stochastic dominance and compare gambles in terms of their inherent and objective riskiness. Our approach may be summed up in the following three basic principles:

1. A gamble $g$ is less risky than a gamble $h$ whenever risk-averse decision-makers are less averse to $g$ than to $h$. 
2. Aversion to a gamble is conveyed by its rejection.

3. Rejection of different gambles should be compared whenever it is substantive, i.e., uniform over a range of decisions.

Putting these together yields:

A gamble $g$ is less risky than a gamble $h$ whenever

$g$ is uniformly rejected less than $h$ by risk-averse decision-makers.

This principle yields two orders-wealth-uniform dominance and utilityuniform dominance-which are complete orders, and moreover equivalent to the Aumann and Serrano (2008) index of riskiness and the Foster and Hart (2009) measure of riskiness, respectively.

The contribution of this paper is twofold: first, in showing how natural and simple modifications allow one to complete the stochastic dominance order, and thus compare any two gambles; and second, in providing a new, ordinal approach to riskiness. Moreover, all this is carried out in one unified and standard framework, which helps provide additional understandings and insights into these concepts and their connections.

The paper is organized as follows. Section II includes the standard setup and preliminaries. The orders on gambles-from stochastic dominance to our two uniform dominance orders-are presented in Section III, together with the main result. In Section IV we discuss various pertinent issues and possible extensions. The proofs, together with some additional results, are relegated to the Appendix.

\section{Preliminaries}

A gamble $g$ is a real-valued random variable with positive expectation and some negative values (i.e., ${ }^{5} \mathbf{E}[g]>0$ and $\mathbf{P}[g<0]>0$ ); for simplicity, assume that $g$ takes finitely many values (cf. Sec. IV $(\mathrm{g})$ ). Let $\mathcal{G}$ denote the collection of all such gambles. For each gamble $g$ in $\mathcal{G}$, denote by $M_{g}:=\max g$ the maximal gain of $g$, and by $L_{g}:=\max (-g)=-\min g$ its maximal loss; $\max |g|=\max \left\{M_{g}, L_{g}\right\}$ is its overall bound. One should view a gamble as the net returns of a risky asset; that is, the values of $g$ represent the possible changes in wealth when $g$ is realized (the positive values of $g$ are gains, and the negative ones, losses).

A (von Neumann and Morgenstern) risk-averse utility function $u$ is a strictly increasing and concave function ${ }^{6} u: \mathbb{R}_{+} \rightarrow \mathbb{R}$. "Risk aversion" is

${ }^{5}$ Let $\mathbf{E}$ and $\mathbf{P}$ denote expectation and probability, respectively. The probability space on which the random variable $g$ is defined is irrelevant; only the distribution of $g$ will matter. We chose to work with random variables $g$ rather than their distributions $G$ for convenience, as $\mathbf{E}[g]$ appears simpler than $\mathbf{E}_{G}[\cdot]$.

${ }^{6}$ Let $\mathbb{R}=(-\infty, \infty)$ denote the set of real numbers, and $\mathbb{R}_{+}=(0, \infty)$ the set of positive numbers. 
represented by the concavity assumption: the utility of a sure outcome of $a$ is always at least as large as the expected utility of a random variable with expectation $a$; i.e., $u(\mathbf{E}[X]) \geq \mathbf{E}[u(X)]$ for any random variable $X$. Let $\mathcal{U}$ denote the collection of all such utility functions $u$.

\section{A. Accepting and Rejecting Gambles}

A decision-maker is characterized by a utility function $u \in \mathcal{U}$ and a wealth level $w>0$. The decision-maker accepts a gamble $g \in \mathcal{G}$ if $\mathbf{E}[u(w+g)]>$ $u(w)$, and rejects $g$ if $\mathbf{E}[u(w+g)] \leq u(w)$; that is, a gamble is accepted if the expected utility from accepting is higher than from staying put, and is rejected otherwise. ${ }^{7}$

REMARK. Since the utility $u(x)$ is not defined for $x \leq 0$, acceptance of $g$ is considered only at wealth levels $w$ such that ${ }^{8} w+g>0$, or $w>$ $L_{g}$. Thus statements such as " $g$ is accepted by $u$ at all $w$ " should be understood to refer to all $w>L_{g}$.

\section{B. Regular Utilities}

We will use two standard assumptions on utility functions propounded by Arrow (1965, lecture 2; 1971, p. 96); these assumptions amount to certain monotonicity relations between decisions and wealth levels. The first is that acceptance increases ${ }^{9}$ with wealth: if $u$ accepts a gamble $g$ at wealth level $w$ then $u$ accepts $g$ also at any higher wealth level $w^{\prime}>w$. The second is that acceptance decreases with relative wealth: scaling up both the gamble and the wealth by the same factor that is greater than 1 decreases acceptance (and thus scaling down by a factor that is less than 1 increases acceptance); that is, if $u$ rejects $g$ at wealth level $w$ then $u$ also rejects ${ }^{10} \lambda g$ at wealth level $\lambda w$ for every $\lambda>1$ (equivalently, if $u$ accepts $g$ at $w$ then $u$ accepts $\lambda g$ at $\lambda w$ for every $0<\lambda<1)$.

For another way to state these conditions, assume ${ }^{11}$ that the utility

${ }^{7}$ The decision in the case of indifference (i.e., when $\mathbf{E}[u(w+g)]=u(w)$ ) does not matter. We could have acceptance instead of rejection, or even leave this undefined; while some of the inequalities in the proofs may change from strict to weak and vice versa, none of the final results are affected.

${ }^{8}$ The inequality $w+g>0$ means that $w+x>0$ for all values $x$ of $g$. It may be convenient to put $u(x):=-\infty$ for $x \leq 0$ (which makes $u$ concave over all $\mathbb{R}$ ), and then for every $w \leq L_{g}$ we have $\mathbf{E}[u(w+g)]=-\infty \leq u(w)$ and $g$ is indeed rejected at such $w$.

9 Recall that "increasing" and "decreasing" should always be understood in the weak sense (i.e, they mean "nondecreasing" and "nonincreasing," respectively); when needed, we will use "strictly" explicitly.

${ }^{10}$ The gamble $\lambda g$ is obtained by multiplying all outcomes of $g$ by the factor $\lambda$ (and leaving the probabilities unchanged).

${ }^{11}$ The differentiability assumptions do not matter, as our concepts and constructs are continuous with respect to pointwise convergence of the utility functions (and the gambles are bounded). 
functions $u \in \mathcal{U}$ are twice continuously differentiable (i.e., of class $C^{2}$ ) and $u^{\prime}(x)>0$ for every $x>0$. The Arrow-Pratt coefficient of Absolute Risk Aversion ("ARA") of $u$ at $x$ is $\rho_{u}(x):=-u^{\prime \prime}(x) / u^{\prime}(x)$, and the coefficient of Relative Risk Aversion ("RRA") is $\tilde{\rho}_{u}(x):=-x u^{\prime \prime}(x) / u^{\prime}(x)=\rho_{u}(x) /(1 / x)$ (see Arrow 1965, 1971; Pratt 1964). The first condition of "acceptance increasing with wealth" corresponds to Decreasing Absolute Risk Aversion ("DARA"): $\rho_{u}$ is a decreasing function of wealth, i.e., $\rho_{u}\left(x^{\prime}\right) \leq \rho_{u}(x)$ for all $x^{\prime}>x$ (see Pratt 1964; Yaari 1969; Dybvig and Lippman 1983). Similarly, the second condition of "acceptance decreasing with relative wealth" corresponds to Increasing Relative Risk Aversion ("IRRA"): $\tilde{\rho}_{u}$ is an increasing function of wealth, i.e., $\tilde{\rho}_{u}\left(x^{\prime}\right) \geq \tilde{\rho}_{u}(x)$ for all $x^{\prime}>x$. Let $\mathcal{U}_{\mathrm{DA}}:=\left\{u \in \mathcal{U}: \rho_{u}(x)\right.$ decreases in $\left.x\right\}$ and $\mathcal{U}_{\mathrm{IR}}:=\left\{u \in \mathcal{U}: \tilde{\rho}_{u}(x)\right.$ increases in $x\}$ denote these two collections of utilities.

Two special families of utilities (that belong to both $\mathcal{U}_{\mathrm{DA}}$ and $\mathcal{U}_{\mathrm{IR}}$ ) are the Constant Absolute Risk Aversion ("CARA") utilities $\bar{v}_{\alpha}$ for $\alpha>0$, where $\bar{v}_{\alpha}(x):=-\exp (-\alpha x)$ (and thus $\rho_{\bar{v}_{\alpha}}(x)=\alpha$ for all $x$ ), and the Constant Relative Risk Aversion ("CRRA") utilities $\tilde{v}_{\gamma}$ for $\gamma \geq 0$, where $\tilde{v}_{\gamma}(x):=$ $x^{1-\gamma} /(1-\gamma)$ for $\gamma \neq 1$ and $\tilde{v}_{1}(x)=\log (x)$ (and thus $\tilde{\rho}_{\tilde{\nu}_{\gamma}}(x)=\gamma$ for all $x>0)$; let $\mathcal{U}_{\mathrm{CA}}:=\left\{\bar{v}_{\alpha}: \alpha>0\right\}$ and $\mathcal{U}_{\mathrm{CR}}:=\left\{\tilde{v}_{\gamma}: \gamma \geq 0\right\}$.

A final requirement imposed on a utility function $u$ will be that no gamble should always be accepted by $u$ : for every $g \in \mathcal{G}$ there is $w>$ $L_{g}$ such that $u$ rejects $g$ at $w$. A utility function $u$ that does not satisfy this condition accepts certain gambles no matter how low the wealth is (which may suggest that $u$ is "less qualified" to attest to the riskiness of gambles, as it is willing to accept too many risks)..$^{12}$ Let $\mathcal{U}_{\mathrm{sr}}$ denote this collection of utility functions ("sr" stands for "some rejection").

Altogether, we will denote by $\mathcal{U}^{*}:=\mathcal{U}_{\mathrm{DA}} \cap \mathcal{U}_{\mathrm{IR}} \cap \mathcal{U}_{\mathrm{sr}}$ the resulting class of utilities; as we will see in Section $\mathrm{C}$ in the Appendix, $u \in \mathcal{U}^{*}$ if and only if $\rho_{u}$ decreases, $\tilde{\rho}_{u}$ increases, and $\lim _{x \rightarrow 0^{+}} u(x)=-\infty$ (the last condition is equivalent to $\left.\inf _{x>0} \tilde{\rho}_{u}(x)=\lim _{x \rightarrow 0^{+}} \tilde{\rho}_{u}(x) \geq 1\right)$. In particular, $\mathcal{U}^{*}$ contains all CRRA utilities $\tilde{v}_{\gamma}$ with RRA coefficient $\gamma \geq 1$, utilities that appear consistent with observed behavior; ${ }^{13}$ it also contains utilities that are CARA from some wealth on. ${ }^{14}$

We emphasize that we chose to work throughout with one collection of utilities, $\mathcal{U}^{*}$, for convenience only; see the discussion in Section IV(d) and (e).

${ }^{12}$ For example, $u(x)=\sqrt{x}$ does not belong to $\mathcal{U}_{\mathrm{sr}}$ (for instance, it always accepts the halfhalf gamble on $\$ 4$ and $-\$ 1)$, whereas both $\log (x)$ and $-1 / x$ do belong to $\mathcal{U}_{\text {sr }}$; see the next paragraph.

${ }^{13}$ See, e.g., Meyer and Meyer $(2005,2006)$ and Palacios-Huerta and Serrano (2006).

${ }^{14}$ Take for example $\hat{v}_{\alpha}(x):=(\log (\alpha x)-1) / e$ for $x \leq 1 / \alpha$ and $\hat{v}_{\alpha}(x):=-\exp (-\alpha x)$ for $x \geq 1 / \alpha$; then $\rho_{\hat{v}_{\alpha}}(x)=1 / x$ for $x \leq 1 / \alpha$ and $\rho_{\hat{v}_{\alpha}}(x)=\alpha$ for $x \geq 1 / \alpha$ and so $\hat{v}_{\alpha} \in \mathcal{U}^{*}$ for each $\alpha>0$. 


\section{Numerical Measures of Riskiness}

How can one quantify the intrinsic riskiness of gambles-that is, assign to each gamble a real number that measures its riskiness? Again, we want to do this in an objective way, independent of any specific decisionmaker. Just as the "return" of the gamble (its expectation) and the "spread" of the gamble (its standard deviation) depend only on the gamble itself (i.e., its distribution: outcomes and probabilities) and are thus objective measures, so the riskiness of the gamble should be.

Two such recent approaches are the "economic" index of riskiness developed by Aumann and Serrano (2008), ${ }^{15}$ and the "operational" measure of riskiness of Foster and Hart (2009). ${ }^{16}$ Although based on quite different considerations, they turn out to be similar in many ways, and to share several useful properties (besides being objective measures), such as monotonicity with respect to (first- and second-degree) stochastic dominance; see Aumann and Serrano (2008), Foster and Hart (2009; Sec. VI.A compares the two approaches), and Foster and Hart (2011).

Formally, for every gamble $g \in \mathcal{G}$ :

- $R^{\mathrm{AS}}(g)$, the Aumann-Serrano index of riskiness of $g$, is given by

$$
\mathbf{E}\left[\exp \left(-\frac{1}{R^{\mathrm{AS}}(g)} g\right)\right]=1
$$

That is, consider the equation $\mathbf{E}[\exp (-\alpha g)]=1$; it has a unique positive solution $\alpha=\alpha^{*}(g)>0$, i.e., ${ }^{17}$

$$
\mathbf{E}\left[\exp \left(-\alpha^{*}(g) g\right)\right]=1,
$$

and then

$$
R^{\mathrm{AS}}(g):=\frac{1}{\alpha^{*}(g)}
$$

(see Aumann and Serrano 2008, theorems A and B).

This means that among the CARA utilities $\bar{v}_{\alpha} \in \mathcal{U}_{\mathrm{CA}}$, the one with coefficient $\alpha=\alpha^{*}(g)$ is always indifferent between accepting and rejecting $g$, whereas all those with a higher absolute risk-aversion coefficient $\alpha>\alpha^{*}(g)$ always reject $g$, and all those with a lower one $\alpha<$ $\alpha^{*}(g)$ always accept $g$; here "always" stands for "at all wealth levels $w$."

\footnotetext{
15 This index was used in the technical report of Palacios-Huerta, Serrano, and Volij (2004); see the footnote on page 810 of Aumann and Serrano (2008).

${ }^{16}$ For a discussion of some of the earlier work, see Sec. VIII in Aumann and Serrano (2008) and Sec. VI.D in Foster and Hart (2009). The reason one is called an "index" and the other a "measure" is explained in Foster and Hart (2009, Sec. VI.A); see also Sec. IV(a) below.

${ }^{17}$ In the insurance risk literature, $\alpha *$ is called the "adjustment coefficient"; see Meilijson (2009).
} 
We can informally say that $1 / R^{\mathrm{AS}}(g)$ is the critical risk aversion level for $g$.

- $R^{\mathrm{FH}}(g)$, the Foster-Hart measure of riskiness of $g$, is given by

$$
\mathbf{E}\left[\log \left(1+\frac{1}{R^{\mathrm{FH}}(g)} g\right)\right]=0 .
$$

That is, consider the equation $\mathbf{E}[\log (r+g)]=\log (r)$; it has a unique positive solution $r=R^{\mathrm{FH}}(g)>L_{g}$, i.e.,

$$
\mathbf{E}\left[\log \left(R^{\mathrm{FH}}(g)+g\right)\right]=\log \left(R^{\mathrm{FH}}(g)\right)
$$

(see Foster and Hart 2009, theorem 1).

That is, $R^{\mathrm{FH}}(g)$ is the wealth level where the CRRA utility $\tilde{v}_{1} \in \mathcal{U}_{\mathrm{CR}}$ with RRA coefficient 1 (i.e., $\tilde{v}_{1}(x)=\log (x)$ ) is indifferent between accepting and rejecting the gamble $g$; at any higher wealth level $w>$ $R^{\mathrm{FH}}(g)$ the utility $\tilde{v}_{1}$ accepts $g$, and at any lower wealth level $w<$ $R^{\mathrm{FH}}(g)$ it rejects $g$. Informally, $R^{\mathrm{FH}}(g)$ is the critical wealth level for $g$ : as shown in Foster and Hart (2009), to avoid decreasing wealth and bankruptcy, $g$ is rejected at any wealth level $w$ below the measure of riskiness $R^{\mathrm{FH}}(g)$ of $g$.

\section{Comparing Gambles}

We start from the well-known stochastic dominance order and proceed to our two new uniform dominance orders, defined in Sections III.C and III.D; the main result is stated in Section III.E.

Let $g$ and $h$ be gambles in $\mathcal{G}$; the objective is to find out when $g$ "dominates" $h$, in the sense that $g$ is less risky than $h$ and thus risk-averse decision-makers are less averse to $g$ than to $h$.

\section{A. Stochastic Dominance}

The first approach is based on "desirability": if every risk-averse decisionmaker prefers $g$ to $h$, this is a clear indication that $g$ is less risky than $h$. This yields the classical comparison known as "second-degree stochastic dominance" ${ }^{18}$ (see Hadar and Russell 1969; Hanoch and Levy

\footnotetext{
18 "Second degree" refers to risk-averse utility functions (i.e., in $\mathcal{U}$ : strictly increasing, and concave), whereas "first degree" refers to utility functions that are just strictly increasing (and not necessarily concave). The second-degree order is thus an extension of the first-degree order: if $g$ first-degree stochastically dominates $h$, then $g$ second-degree stochastically dominates $h$ (nevertheless, many authors restrict "second-degree" only to the additional comparisons that go beyond the first-degree order, and then deal only with pairs of gambles with identical expectations).

Since risk and risk aversion are of the essence in this paper, the second-degree order is the relevant one, and so "stochastic dominance" will always be of the second degree.
} 
1969; Rothschild and Stiglitz 1970, 1971; Machina and Rothschild 2008): $g$ stochastically dominates $h$, which we denote ${ }^{19} g \geq_{\mathrm{S}} h$ ("S" stands for "Stochastic"), if the expected utility that $g$ yields is always at least as large as that of $h$; i.e., for every $u \in \mathcal{U}$ and every $^{20} w$,

$$
\mathbf{E}[u(w+g)] \geq \mathbf{E}[u(w+h)] .
$$

Thus, given the choice between $g$ and $h$, every risk-averse decision-maker always prefers $g$ to $h$.

As is well known (see for instance the above references), $g$ seconddegree stochastically dominates $h$ if and only if there are $g^{\prime}, h^{\prime}, h^{\prime \prime}$ that are all defined on the same probability space, such that the following holds: $g^{\prime} \geq h^{\prime}$ (i.e., in each state the realization of $g^{\prime}$ is no less than the realization of $h^{\prime}$ ); $h^{\prime \prime}$ is obtained from $h^{\prime}$ by a sequence of meanpreserving spreads (which replace an outcome $x$ of $h^{\prime}$ with a lottery whose expectation equals $x) ; g^{\prime}$ has the same distribution as $g$; and $h^{\prime \prime}$ has the same distribution as $h$. That is, $g$ has higher gains, or lower losses, or fewer lotteries than $h$ (more precisely, this statement applies to $g^{\prime}$ and $h^{\prime \prime}$ ).

Stochastic dominance yields a clear and uncontroversial order on gambles; however, it is a very partial order, ${ }^{21}$ with most pairs of gambles $g$ and $h$ being incomparable: neither stochastically dominates the other.

\section{B. Extending Stochastic Dominance}

As discussed in the Introduction, we first weaken the requirement in (4) by considering decision-makers who are allowed to choose whether to accept or reject gambles. Thus we say that $g$ acceptance dominates ${ }^{22} h$, denoted $g \geq_{\mathrm{A}} h$ ("A" stands for "Acceptance"), if $g$ is accepted more than $h$ (and so is rejected less than $h$ ) by all risk-averse decision-makers; i.e., for every $u \in \mathcal{U}$ and every $w>0$,

$$
\text { if } g \text { is rejected by } u \text { at } w
$$

then $h$ is rejected by $u$ at $w$;

${ }^{19}$ Recall that we deal throughout with the weak versions of the orders.

${ }^{20}$ At this point the reader may ask why is the wealth $w$ used at all, as (4) is equivalent to $\mathbf{E}[u(g)] \geq \mathbf{E}[u(h)]$ for all strictly increasing and concave $u$ (take $\tilde{u}(x):=u(w+x)$ ). Although this is indeed irrelevant for stochastic dominance, it will become significant for our uniform dominance orders.

${ }^{21}$ An order (sometimes called "preorder" or "partial order") $\geq_{*}$ is a binary relation that is reflexive (i.e., $g \geq_{*} g$ for any $g$ ) and transitive (i.e., $g \geq_{*} h$ and $h \geq_{*} k$ imply $g \geq_{*} k$, for any $g, h, k)$. It is a complete (sometimes called "linear") order if every pair $g, h$ can be compared (i.e., either $g \geq_{*} h$ or $h \geq_{*} g$ for any $\left.g, h\right)$.

${ }^{22}$ A more apt, if cumbersome, name would be "acceptance stochastic dominance" (the term "stochastic" refers to the fact that only the distributions of the gambles-i.e., values and probabilities-matter). Since all the orders in this paper are "stochastic," we will drop this word for simplicity (except in the case of the original stochastic dominance of Sec. III.A). 
equivalently,

$$
\mathbf{E}[u(w+g)] \leq u(w) \quad \text { implies } \quad \mathbf{E}[u(w+h)] \leq u(w) .
$$

While acceptance dominance extends stochastic dominance (i.e., $g \geq_{\mathrm{S}} h$ implies $g \geq_{\mathrm{A}} h$, since (4) implies (6)), it is still only a partial order, and for general gambles $g$ and $h$ neither one will acceptance dominate the other. ${ }^{23}$ As argued in the Introduction, the reason is that the requirement (5) for each and every instance, i.e., for every $u$ and $w$, is too strong a condition. The values of $g$ and $h$ may be very different, and then it would be hard to deduce from the fact that a certain utility function $u$ rejects $g$ at a certain wealth level $w$ that exactly the same occurs for $h$.

This suggests that one may seek stronger evidence of "aversion" to $g$ before requiring that the same hold for $h$. Thus, rather than a single instance of rejection, one should consider a whole range of rejections: "uniform rejection." Since an acceptance/rejection decision is characterized by a utility function $u$ and a wealth level $w$, there are two natural ways of doing so: uniformly over the wealth levels, and uniformly over the utility functions. These two "uniform dominance" orders will be the subject of the next two sections. For simplicity we will from now on restrict ourselves to regular utility functions $u \in \mathcal{U}^{*}$, which guarantees that the uniform conditions do not become vacuous (see however Sec. $\mathrm{IV}(\mathrm{d})$ and (e)).

\section{Wealth-Uniform Dominance}

We start with "wealth-uniformity": say that a gamble $g$ is wealth-uniformly rejected by $u$ if $g$ is rejected by $u$ at all wealth levels $w$.

Our first uniform order is defined as follows: a gamble $g \in \mathcal{G}$ wealthuniformly dominates a gamble $h \in \mathcal{G}$, denoted $g \geq_{\mathrm{wU}} h$ ("WU" stands for "Wealth-Uniform"), if $h$ is wealth-uniformly rejected more than $g$; i.e., for every utility $u \in \mathcal{U}^{*}$,

$$
\text { if } g \text { is rejected by } u \text { at all } w>0
$$

then $h$ is rejected by $u$ at all $w>0$.

That is, if $g$ is wealth-uniformly rejected, then so is $h$.

Formally, $g \geq_{\mathrm{wU}} h$ if for every $u \in \mathcal{U}^{*}$

$$
\begin{gathered}
(\mathbf{E}[u(w+g)] \leq u(w) \text { for all } w>0) \\
\text { implies }(\mathbf{E}[u(w+h)] \leq u(w) \text { for all } w>0) .
\end{gathered}
$$

${ }^{23}$ For example, let $g \in \mathcal{G}$ be the gamble where one gains 20 or loses 10 with equal probabilities of $1 / 2,1 / 2$, and $h \in \mathcal{G}$ the gamble where one gains 50 with probability $2 / 3$ and loses 20 with probability $1 / 3$; then $u_{1}(x)=\log (x)$ at $w_{1}=21$ accepts $g$ and rejects $h$, whereas $u_{2}(x)=-1 / x$ at $w_{2}=39$ rejects $g$ and accepts $h$. 
This captures the idea that a less risky gamble is rejected less often; however, only when the rejection of $g$ occurs at all wealth levels-a strong premise-do we require that the same hold for $h$.

It is immediate to see that wealth-uniform dominance is a partial order (reflexive and transitive), and that it extends stochastic dominance: $g \geq_{\mathrm{s}} h$ implies $g \geq_{\mathrm{wU}} h$ (recall (4)).

\section{Utility-Uniform Dominance}

We come now to "utility-uniformity": say that a gamble $g$ is utility-uniformly rejected at wealth level $w$ if $g$ is rejected by all utility functions $u \in \mathcal{U}^{*}$ at $w$.

Our second uniform dominance order is defined as follows: a gamble $g \in \mathcal{G}$ utility-uniformly dominates a gamble $h \in \mathcal{G}$, denoted $g \geq_{\mathrm{UU}} h$ ("UU" stands for "Utility-Uniform"), if $h$ is utility-uniformly rejected more than $g$; i.e., for every wealth level $w>0$,

$$
\text { if } g \text { is rejected by all } u \in \mathcal{U}^{*} \text { at } w
$$

then $h$ is rejected by all $u \in \mathcal{U}^{*}$ at $w$.

That is, if $g$ is utility-uniformly rejected, then so is $h$.

Formally, $g \geq_{\mathrm{UU}} h$ if for every $w>0$

$$
\begin{gathered}
\left(\mathbf{E}[u(w+g)] \leq u(w) \text { for all } u \in \mathcal{U}^{*}\right) \\
\text { implies }\left(\mathbf{E}[u(w+h)] \leq u(w) \text { for all } u \in \mathcal{U}^{*}\right) .
\end{gathered}
$$

This is another way to capture the idea that a less risky gamble is rejected less often; but now, only when the rejection of $g$ at a certain wealth level is by all decision-makers do we require the same for $h$. The fact that this is a strong premise makes the requirement reasonable.

It is immediate to see that utility-uniform dominance is also a partial order (reflexive and transitive), and that it extends stochastic dominance: $g \geq_{\mathrm{S}} h$ implies $g \geq_{\mathrm{UU}} h$ (recall (4)).

\section{E. The Result}

We now state our main result.

\section{Theorem 1.}

(i) Wealth-uniform dominance $\geq_{\mathrm{wU}}$ is a complete order on $\mathcal{G}$ that extends stochastic dominance. Moreover, for any two gambles $g$ and $h$ in $\mathcal{G}$,

$$
g \geq_{\mathrm{wU}} h \text { if and only if } R^{\mathrm{AS}}(g) \leq R^{\mathrm{AS}}(h),
$$

where $R^{\mathrm{AS}}$ denotes the Aumann-Serrano index of riskiness. 
(ii) Utility-uniform dominance $\geq_{\mathrm{UU}}$ is a complete order on $\mathcal{G}$ that extends stochastic dominance. Moreover, for any two gambles $g$ and $h$ in $\mathcal{G}$,

$$
g \geq_{\mathrm{UU}} h \text { if and only if } R^{\mathrm{FH}}(g) \leq R^{\mathrm{FH}}(h),
$$

where $R^{\mathrm{FH}}$ denotes the Foster-Hart measure of riskiness.

Thus, while it may appear from their definition that the uniform dominance orders, just like stochastic and acceptance dominance, are only partial orders (i.e., not every pair of gambles may be compared), they both turn out to be complete: for any $g, h \in \mathcal{G}$, either $g \geq_{\mathrm{WU}} h$ or $h \geq_{\mathrm{wU}} g$; and, either $g \geq_{\mathrm{UU}} h$ or $h \geq_{\mathrm{UU}} g$.

Moreover, (7) says that $\geq_{w U}$ is equivalent to the order induced by the Aumann-Serrano index $R^{\mathrm{AS}}$; in other words, $R^{\mathrm{AS}}$ represents the wealthuniform dominance order. ${ }^{24}$ Thus $R^{\mathrm{AS}}$ is uniquely determined by wealthuniform dominance $\geq_{\mathrm{WU}}$ up to monotonic transformations. ${ }^{25}$ Similarly, (8) says $R^{\mathrm{FH}}$ represents the utility-uniform dominance order $\geq_{\mathrm{UU}}$, and so $\geq_{\mathrm{UU}}$ determines $R^{\mathrm{FH}}$ uniquely up to monotonic transformations.

In summary, we started by defining two simple "riskiness" orders on gambles - wealth-uniform dominance and utility-uniform dominanceand then established that these orders are uniquely (up to monotonic transformations) represented by the Aumann-Serrano index and the Foster-Hart measure, respectively. This parallels the standard route of decision theory and consumer theory, which starts with an order on outcomes (a "preference" order) and then represents it by a numerical index (a "utility function"). In our approach the two uniform dominance orders yield the AS-index and the FH-measure directly, without any further postulates or constructions (see Sec. IV(a) and (1)).

The proof of theorem 1 is relegated to Sections $B$ and $C$ in the Appendix.

\section{Discussion}

This section discusses a number of issues and presents some possible extensions.

(a) Ordinal approach to riskiness.-The approach to riskiness in this paper is ordinal, in the sense that we compare gambles (and the end

${ }^{24}$ Just as a consumer's utility function represents his preference order on commodity bundles (in our case the order $\geq_{\mathrm{WU}}$ and the riskiness function $R^{\mathrm{AS}}$ go in opposite directions: WU-dominance corresponds to lower riskiness).

${ }^{25}$ That is, a real-valued function $Q$ on $\mathcal{G}$ represents the $\geq_{\mathrm{wU}}$ order if and only if $Q$ is ordinally equivalent to $R^{\mathrm{AS}}$ : there exists a strictly increasing function $\phi$ such that $Q(g)=\phi\left(R^{\mathrm{AS}}(g)\right)$ for all $g \in \mathcal{G}$. If we require in addition that $Q$ be positively homogeneous of degree one, then $Q$ is a constant multiple of $R^{\mathrm{AS}}$ : there exists a constant $c>0$ such that $Q(g)=c R^{\mathrm{AS}}(g)$ for all $g \in \mathcal{G}$. Compare this to the main results of Aumann and Serrano (2008, theorems D and A). 
results are complete orders), whereas the numerical measures of $\mathrm{Au}$ mann and Serrano (2008) and Foster and Hart (2009) may be viewed as cardinal, as they associate a numerical value to each gamble ${ }^{26}$ theorem 1 provides the connections.

The Foster-Hart measure of riskiness has a clear (and "operational") interpretation: the critical wealth levels below which accepting gambles may lead in the long run to decreasing wealth and bankruptcy; therefore, applying a monotonic transformation to it may not make much sense. In contrast, the derivation and interpretation of the AumannSerrano index of riskiness-which have to do with the critical risk-aversion coefficient-are less conclusive in pinning down this index within the class of all its monotonic, or linear, transformations (cf. theorems D and A in Aumann and Serrano 2008; see also Sec. IV.C there). In a sense, the Aumann-Serrano index seems to be more of an ordinal concept, whereas the Foster-Hart measure is more cardinal.

Finally, note that the Foster and Hart (2009) approach applies to general setups that go beyond utility and expected utility.

(b) Duality between wealth and utility.-As noted already in Foster and Hart (2009, Sec. VI.A), the constructions of the Aumann-Serrano index and the Foster-Hart measure exhibit an interesting duality between wealth and utility. The approach of the present paper further underscores this duality: wealth-uniformity yields the critical utility (and the AS-index), and utility-uniformity yields the critical wealth (and the FHmeasure).

(c) Status quo.-A basic ingredient that enabled us to go beyond the classical stochastic dominance is the status quo, i.e., the current wealth level (indeed, stochastic dominance looks only at the final outcomes). Allowing the decision-makers to reject gambles-rather than just choose which one they prefer-yields additional comparisons between gambles (even before going to the uniform dominance orders). For example, we will show in $(\mathrm{j})$ below that all risk-averse decision-makers reject $\lambda g$ more often than $g$, for any gamble $g \in \mathcal{G}$ and any factor $\lambda>1$; this is a "universal" property that lies below the radar of stochastic dominance (because the status quo and rejection are not seen there).

The idea of status quo together with acceptance and rejection is of course not new (see, e.g., Yaari 1969), and it is already embodied in the Aumann and Serrano (2008) and Foster and Hart (2009) approaches. More generally, the relevance and significance of the status quo have been pointed out in many setups, theoretical and behavioral (e.g., Kahneman and Tversky 1979, Rabin 2000, and many others).

(d) Regular utilities.-We have chosen to use one class of utilities,

${ }^{26}$ In parallel to decision theory: preference orders (ordinal) vs. utility functions (cardinal). 
$\mathcal{U}^{*}$, for both wealth-uniform and utility-uniform dominance; this is more elegant and makes the comparisons clearer. However, as some of the restrictions in $\mathcal{U}^{*}$ may appear at times controversial, ${ }^{27}$ we will show in the Appendix (see the remarks at the end of Secs. B and C) that we can replace $\mathcal{U}^{*}$ by other classes of utilities; for instance, by $\mathcal{U}_{\mathrm{DA}}$ for wealthuniform dominance, and by $\mathcal{U}_{\mathrm{IR}} \cap \mathcal{U}_{\mathrm{sr}}$ or $\mathcal{U}_{\mathrm{CR}} \cap \mathcal{U}_{\mathrm{sr}}$ for utility-uniform dominance. Note here yet another "duality": of Arrow's two conditions (recall Sec. III.B), the one that acceptance increases with wealth is used for $\geq_{w U}$ and $R^{\mathrm{AS}}$, whereas the other one that acceptance decreases with relative wealth is used for $\geq_{\mathrm{UU}}$ and $R^{\mathrm{FH}}$.

(e) General utilities.-Without some regularity assumptions on the acceptance/rejection decisions, the uniformity requirements used in our orders become vacuous (and so one does not go beyond acceptance dominance). Indeed, a utility function whose risk-aversion coefficient oscillates up and down ${ }^{28}$ will lead to nonmonotonic decisions that oscillate between rejection and acceptance as the wealth changes-see proposition 4 in the Appendix (for an explicit example, see Sec. 5.3 in Hart 2009); but how reasonable are decision-makers that, say, accept a gamble $g$ at wealth $\$ 1,000$ and at wealth $\$ 1,002$, but reject it at wealth $\$ 1,001$ ?

(f) Comparing the two uniform orders.-An interesting issue is understanding the similarities and the differences between the two uniform dominance orders. Since the two orders extend stochastic dominance (and acceptance dominance), they agree on these comparisons. Also, they become more and more similar as the riskiness of the gambles increases; this follows from proposition 4 in Section VI.A of Foster and Hart (2009). Beyond that, are there other interesting cases where the two orders agree?

A related question is which measure of riskiness to use, the AumannSerrano index or the Foster-Hart measure? What we learn from the present paper is, on the one hand, that the two measures stand on parallel foundations, which suggests that neither is always preferable to the other. On the other hand, we also learn that the Aumann-Serrano index may be better suited when the utility and the risk aversion matter while the wealth is unknown or less significant, and that the FosterHart measure may be better suited when the wealth matters while the utility and the risk aversion are unknown or less significant. But since, after all, summarizing a whole distribution of a gamble into one single

\footnotetext{
${ }^{27}$ Increasing relative risk-aversion $\left(\mathcal{U}_{\mathrm{IR}}\right)$ is perhaps the most disputed assumption; decreasing absolute risk-aversion $\left(\mathcal{U}_{\mathrm{DA}}\right)$ and constant relative risk-aversion $\left(\mathcal{U}_{\mathrm{CR}}\right)$ are less so (see, e.g., Meyer and Meyer 2005, 2006).

${ }^{28}$ For some of our results it suffices to require monotonic decisions from some wealth on.
} 
number can never tell the whole story, having more than one measure can only help. ${ }^{29}$ Additional studies will hopefully clarify this further.

(g) The class of gambles.-To avoid inessential technical issues, we have maintained throughout the assumption that each gamble takes only finitely many values. It should not be difficult to relax this and replace it with, say, boundedness (at least from below).

Our gambles $g \in \mathcal{G}$ have positive expectation and take some negative values; this is the interesting case. Indeed, a random variable $f$ with $\mathbf{E}[f] \leq 0$ is rejected by every risk-averse decision-maker, and a (nontrivial) random variable $k \geq 0$ is accepted by every decision-maker, and so any gamble $g$ in $\mathcal{G}$ acceptance dominates $f$ and is acceptance dominated by $k$, i.e., $k \geq_{\mathrm{A}} g \geq_{\mathrm{A}} f$ for all ${ }^{30} g \in \mathcal{G}$.

(h) Beyond expected utility.-This paper (as well as Aumann-Serrano 2008 and Foster-Hart 2009) deals with pure risk in the standard von Neumann and Morgenstern expected utility setup. It would be interesting to go beyond that and consider more general setups-such as subjective probability, uncertainty, and various non-expected-utility models-and try to capture universal notions of "more risky" and/or "more uncertain."

(i) Characterizations of dominance.-First- and second-degree stochastic dominance have equivalent characterizations (in terms of lower values and mean-preserving spreads). Restricting the utilities to some of the classes in this paper $\left(\mathcal{U}_{\mathrm{DA}}\right.$, or $\mathcal{U}_{\mathrm{IR}}$, or $\left.\mathcal{U}^{*}\right)$ may affect the stochastic order, ${ }^{31}$ and it would be of interest to obtain appropriate characterizations (cf. Whitmore 1970 and the survey of Levy 1992); the same applies to acceptance dominance.

(j) Acceptance dominance.-To see that acceptance dominance in fact goes beyond stochastic dominance, take any gamble $g \in \mathcal{G}$ and put $h:=\lambda g$ where $\lambda>1$ (i.e., multiply all the outcomes of $g$ by the constant factor $\lambda>1$ ). Although $g$ cannot stochastically dominate $h$ (for instance, because $\mathbf{E}[g]<\mathbf{E}[h]=\lambda \mathbf{E}[g]$ ), it turns out that $g$ acceptance dominates $h$, i.e., $g \geq_{\mathrm{A}} h$. Indeed, for every concave function $u$ we have

$$
u(w+x) \geq(1 / \lambda) u(w+\lambda x)+(1-1 / \lambda) u(w),
$$

and so

$$
\mathbf{E}[u(w+g)] \geq(1 / \lambda) \mathbf{E}[u(w+h)]+(1-1 / \lambda) u(w) .
$$

Therefore (6) is satisfied: if $u(w) \geq \mathbf{E}[u(w+g)]$-i.e., $g$ is rejected-then necessarily $u(w) \geq \mathbf{E}[u(w+h)]$-i.e., $h$ is also rejected.

A precise characterization of acceptance dominance is provided in

${ }^{29}$ In addition, it may make various "manipulations" harder; see for instance the discussion in Foster and Hart (2011, Sec. 7(b)).

${ }^{30}$ One may thus define the riskiness of these $f$ and $k$ to be $\infty$ and 0 , respectively.

31 In the same way that adding concavity takes us from first-degree to second-degree stochastic dominance. 
Hart (2010, App. A.1): it amounts to stochastic dominance between "dilutions" of the given gambles, where "diluting" a gamble means taking it with a probability that may be less than one.

(k) Wealth-bounded dominance.-Acceptance dominance requires that if $g$ is rejected by $u$ at $w$ then $h$ is also rejected by $u$ at $w$; wealth-uniform dominance derives the same conclusion (that $h$ is rejected by $u$ at $w$ ), but from a much stronger premise (that $g$ is rejected by $u$ at all $w^{\prime}$ ). One may also consider some middle ground between the rejection of $g$ at a single wealth level in (5) and its rejection at all wealth levels in [WU]: rejection at a certain range of wealths.

Indeed, the premise in (5) that $g$ is rejected by $u$ at $w$ tells us very little about the values of $u$ outside the interval $[w+\min g, w+$ $\max g]=\left[w-L_{g}, w+M_{g}\right]$. But $w+h$ may well have outcomes that are far away from this interval, which explains why it is hard, except in special cases, to deduce that every $u$ that rejects $g$ at $w$ also rejects $h$ at $w$ (this is why acceptance dominance is only a partial order). It also suggests that, in order to deduce that $h$ is rejected at $w$, one may want to strengthen the premise on $g$ and require that $g$ be rejected not just at $w$ itself, but also at all wealth levels in a certain interval around $w-$ an interval that is determined by (the ranges of outcomes of) $g$ and $h$..

We thus introduce another order: a gamble $g \in \mathcal{G}$ wealth-boundedly dominates a gamble $h \in \mathcal{G}$, denoted $g \geq_{\mathrm{WB}} h$ ("WB" stands for "WealthBounded"), whenever there exists a bound $b \equiv b(g, h)<\infty$ (that depends only on $g$ and $h$ ) such that for every utility $u \in \mathcal{U}^{*}$ and wealth $w>0$,

$$
\text { if } g \text { is rejected by } u \text { at all } w^{\prime} \text { with }\left|w^{\prime}-w\right| \leq b
$$

then $h$ is rejected by $u$ at $w$.

We emphasize that the bound $b$ depends only on the two gambles $g$ and $h$, but applies to all utility functions $u \in \mathcal{U}^{*}$ and all wealth levels $w>$ 0 .

While wealth-bounded dominance $\geq_{\mathrm{WB}}$ is clearly a partial order lying between $\geq_{A}$ and $\geq_{W U}$, it is in fact equivalent to the latter, i.e., to wealthuniform dominance; this is proved in Section B of the Appendix (proposition 5), where we also show that one can always take $b(g, h)=$ $L_{g}+M_{h} \leq \max |g|+\max |h|$, and so $b$ is indeed of the same order of magnitude as the outcomes of the two gambles. ${ }^{32}$

(l) The duality axiom and the Aumann-Serrano index.-The approach of Aumann and Serrano (2008) is based on their duality axiom. Though on the face of it this postulate seems very reasonable, on closer inspection it turns out to be relatively complex, and its rationale not entirely straightforward. In particular, it involves two decision-makers and the index itself (besides the two gambles that are compared). In contrast,

${ }^{32}$ See Hart (2010, Sec. 4.1) for further details on $\geq_{\mathrm{WB}}$. 
wealth-uniform dominance involves only one decision-maker. All this is discussed in Hart (2010, Secs. 4.2 and A.5), where we analyze the duality axiom and indicate how the approach of the present paper seems to capture the Aumann-Serrano riskiness in a starker and more basic form; indeed, we show how at the basis of the duality axiom lies wealth-uniform dominance, ${ }^{33}$ and so using this order directly simplifies and streamlines the whole approach. ${ }^{34}$

\section{Appendix}

The Appendix contains the proofs and some additional results. ${ }^{35}$

\section{A. Acceptance, Rejection, and Risk Aversion}

From now on we assume that $u \in \mathcal{U}$ is twice continuously differentiable, i.e., of class $C^{2}$, and $u^{\prime}(x)>0$ for every $x>0$. Recall that the Arrow-Pratt risk-aversion coefficient $\rho \equiv \rho_{u}$ of $u$ is given by $\rho(x)=-u^{\prime \prime}(x) / u^{\prime}(x)$ for every $x$. The basic result we will use is that a higher risk-aversion coefficient yields a concave transformation of the utility function, and thus more rejection (cf. Arrow 1965, 1971 and Pratt 1964 - see in particular theorem 1 there) ${ }^{36}$ Specifically:

Proposition 2. Let $u_{1}, u_{2} \in \mathcal{U}$ be two utility functions with absolute riskaversion coefficients $\rho_{1}$ and $\rho_{2}$, respectively, and let $I \subset(0, \infty)$ be an interval ${ }^{37}$ where $\rho_{1}(x) \geq \rho_{2}(x)$ for every $x \in I$. Then for every $w>0$ and $g \in \mathcal{G}$ such that $w+g \subset I$, if $u_{2}$ rejects $g$ at $w$ then $u_{1}$ rejects $g$ at $w$.

Proof. Let $\psi$ be such that $u_{1}=\psi \circ u_{2}$; then $\psi$ is strictly increasing (since $u_{1}$ and $u_{2}$ are such), and concave (since for every $x \in I$ we have $\psi^{\prime}\left(u_{2}(x)\right)=$ $u_{1}^{\prime}(x) / u_{2}^{\prime}(x)$, hence

$$
\left(\log \psi^{\prime}\left(u_{2}(x)\right)\right)^{\prime}=\left(\log u_{1}^{\prime}(x)\right)^{\prime}-\left(\log u_{2}^{\prime}(x)\right)^{\prime}=-\rho_{1}(x)+\rho_{2}(x) \leq 0,
$$

and so $\left.\psi^{\prime \prime} \leq 0\right)$.

Therefore $\mathbf{E}\left[u_{2}(w+g)\right] \leq u_{2}(w)$ implies

$$
\mathbf{E}\left[u_{1}(w+g)\right]=\mathbf{E}\left[\psi\left(u_{2}(w+g)\right)\right] \leq \psi\left(\mathbf{E}\left[u_{2}(w+g)\right]\right) \leq \psi\left(u_{2}(w)\right)=u_{1}(w)
$$

${ }^{33}$ The research program of the present paper originated from this observation; see Hart (2009).

${ }^{34}$ Moreover, the additional assumptions of Aumann and Serrano (2008)-homogeneity (in their theorem A), or continuity together with monotonicity with respect to first-order stochastic dominance (in their theorem D) - are no longer needed in the wealth-uniform dominance approach (see Sec. X.N of Aumann and Serrano 2008 for counterexamples without these additional conditions).

${ }^{35}$ Some of the arguments below are standard; insofar as they are short enough, we have preferred to provide self-contained proofs rather than refer the reader to various other sources.

${ }^{36}$ One may easily prove that the converse-which is not needed in the present paperalso holds: a utility function that always rejects more than another utility function must be a concave transformation of it, and thus have a higher risk-aversion coefficient.

${ }^{37}$ The interval $I$ can be open or closed at either end, and its upper end can be $\infty$. As usual, $w+g \subset I$ means that $w+x \in I$ for every value $x$ of $g$. 
(the concavity of $\psi$ was used in the first inequality, and the monotonicity of $\psi$ and the assumption on $u_{2}$ in the second). QED

Recall that, for $\alpha>0$, the CARA utility $\bar{v}_{\alpha} \in \mathcal{U}_{\mathrm{CA}}$ satisfies $\rho_{\bar{\nu}_{\alpha}}(w)=\alpha$ for every $w$. The definition (1) of $\alpha^{*} \equiv \alpha^{*}(g)$ implies that for every $w$ we have $\mathbf{E}\left[\bar{v}_{\alpha^{*}}(w+g)\right]=\bar{v}_{\alpha^{*}}(w)$. Therefore

Lemma 3. Let $g \in \mathcal{G}$ and $\bar{v}_{\beta} \in \mathcal{U}_{\mathrm{CA}}$. If $\beta<\alpha^{*}(g)$ then $\bar{v}_{\beta}$ accepts $g$ at all $w>$ $L_{g}$, and if $\beta \geq \alpha^{*}(g)$ then $\bar{v}_{\beta}$ rejects $g$ at all $w$.

Proof. Use proposition 2, or direct computation. QED

Proposition 4. Let $u \in \mathcal{U}, g \in \mathcal{G}$, and $w>L_{g}$, and put $I:=\left[w-L_{g}\right.$, $w+$ $\left.M_{g}\right] \equiv[w+\min g, w+\max g]$. Then:

(i) If $\rho_{u}\left(w^{\prime}\right) \geq \alpha^{*}(g)$ for every $w^{\prime} \in I$ then $u$ rejects $g$ at $w$.

(ii) If $\rho_{u}\left(w^{\prime}\right)<\alpha^{*}(g)$ for every $w^{\prime} \in I$ then $u$ accepts $g$ at $w$.

(iii) If $u$ rejects $g$ at $w$ then there exists $w^{\prime} \in I$ such that $\rho_{u}\left(w^{\prime}\right) \geq \alpha^{*}(g)$.

(iv) If $u$ accepts $g$ at $w$ then there exists $w^{\prime} \in I$ such that $\rho_{u}\left(w^{\prime}\right)<\alpha^{*}(g)$.

Proof. We will prove only (i) and (ii), since (iv) is equivalent to (i) and (iii) is equivalent to (ii). Put $\alpha^{*} \equiv \alpha^{*}(g)$.

(i) Since $\bar{v}_{\alpha^{*}}$ rejects $g$ at $w$ (by lemma 3) and $\rho_{\bar{v}_{\alpha^{*}}}\left(w^{\prime}\right)=\alpha^{*}$ for every $w^{\prime}$, proposition 2 with $u_{1}=u$ and $u_{2}=\bar{v}_{\alpha^{*}}$ implies that $u$ rejects $g$ at $w$.

(ii) Let $\beta:=\max _{w^{\prime} \in I} \rho_{u}\left(w^{\prime}\right)$; then $0<\beta<\alpha^{*}$ (use the continuity of $\rho_{u}$ and $w-L_{g}>0$ ). Since $\bar{v}_{\beta}$ accepts $g$ at $w$ (by lemma 3 ), applying proposition 2 with $u_{1}=\bar{v}_{\beta}$ and $u_{2}=u$ implies that $u$ accepts $g$ at $w$. QED

Proposition 4 is essentially (4.3.2) of Aumann and Serrano (2008); we have proved it here directly for completeness (their arguments are slightly more elaborate).

\section{B. Wealth-Uniform Dominance}

In this section we will prove theorem 1(i) together with the following proposition on wealth-bounded dominance (see Sec. IV $(\mathrm{k})$ ).

Proposition 5. Wealth-uniform dominance and wealth-bounded dominance are equivalent: $g \geq_{\mathrm{wU}} h$ if and only if $g \geq_{\mathrm{WB}} h$, for every $g, h$ in $\mathcal{G}$.

Proof of theorem 1(i) and proposition 5. First, we claim that

$$
g \geq_{\mathrm{wU}} h \text { implies } \alpha^{*}(g) \geq \alpha^{*}(h) .
$$

Indeed, if $\alpha^{*}(g)<\alpha^{*}(h)$ then put $\beta:=\alpha^{*}(g)$ and let $u=\hat{v}_{\beta} \in \mathcal{U}^{*}$ be given by $\rho_{u}(x):=\max \{1 / x, \beta\}$ (see footnote 14). Since $\rho_{u}(x) \geq \beta=\alpha^{*}(g)$ for every $x>0$, proposition 4(i) implies that $u$ rejects $g$ at all $w$; since $\rho_{u}(x)=\beta<\alpha^{*}(h)$ for every $x \geq 1 / \beta$, proposition 4(ii) implies that $u$ accepts $h$ at all $w$ such that $w-L_{h} \geq$ $1 / \beta$, which contradicts $g \geq_{\mathrm{wU}} h$.

Second, we claim that

$$
\alpha^{*}(g) \geq \alpha^{*}(h) \text { implies } g \geq_{\mathrm{WB}} h \text { with } b=L_{g}+M_{h} \text {. }
$$

Indeed, if $\alpha^{*}(g) \geq \alpha^{*}(h)$ and $g$ is rejected by $u$ at $w+b$, then there exists $w^{\prime} \in$ $\left[w+b-L_{g}, w+b+M_{g}\right]$ such that $\rho_{u}\left(w^{\prime}\right) \geq \alpha^{*}(g) \geq \alpha^{*}(h)$ (the first inequality by proposition 4(iii), the second by assumption), and so $\rho_{u}\left(w^{\prime}\right) \geq \alpha^{*}(h)$ for every $w^{\prime} \leq w+b-L_{g}$ (since $\rho_{u}$ is decreasing); for $b=L_{g}+M_{h}$ we have $w+b-L_{g}=$ 
$w+M_{h}$, which implies that $h$ is rejected at $w$ (by proposition 4(i)) —and we have thus proved (A1).

Since $g \geq_{\mathrm{WB}} h$ implies $g \geq_{\mathrm{WU}} h$ (see Sec. IV $(\mathrm{k})$ ), altogether we have obtained

$$
g \geq_{\mathrm{WU}} h \text { if and only if } g \geq_{\mathrm{WB}} h \text { if and only if } \alpha^{*}(g) \geq \alpha^{*}(h) ;
$$

recalling (2) completes the proof. QED

Remarks. (1) (A1) shows that one can take

$$
b \equiv b(g, h)=L_{g}+M_{h} \leq \max |g|+\max |h|
$$

as the bound in $[\mathrm{WB}]$.

(2) The proofs above show that for the wealth-uniform results one can replace $\mathcal{U}^{*}$ with any collection of utilities $\mathcal{U}^{* \mathrm{w}} \subset \mathcal{U}$ that satisfies: (i) $\mathcal{U}^{* \mathrm{w}} \subset \mathcal{U}_{\mathrm{DA}}$; and (ii) for every $\beta>0$ there is $u \in \mathcal{U}^{* \mathrm{~W}}$ such that $\inf _{x>0} \rho_{u}(x)=\lim _{x \rightarrow \infty} \rho_{u}(x)=\beta$. In particular, one may take $\mathcal{U}^{* \mathrm{~W}}=\mathcal{U}_{\mathrm{DA}}$.

(3) An additional characterization of wealth-uniform dominance in terms of "rejection classes" that follows from the results above is provided in Hart (2010, proposition 12).

\section{Utility-Uniform Dominance}

We start by characterizing $\mathcal{U}_{\text {sr }}$.

Proposition 6. Let $u \in \mathcal{U}$ and put $u\left(0^{+}\right):=\lim _{x \rightarrow 0^{+}} u(x) .^{38}$ Then:

(i) $u\left(0^{+}\right)=-\infty$ if and only if for every $g \in \mathcal{G}$ there is $\delta>0$ such that $g$ is rejected by $u$ at all $w \in\left(L_{g}, L_{g}+\delta\right)$.

(ii) $u\left(0^{+}\right)>-\infty$ if and only if there is $g \in \mathcal{G}$ and $\delta>0$ such that $g$ is accepted by $u$ at all $w \in\left(L_{g}, L_{g}+\delta\right)$.

Proof. If $u\left(0^{+}\right)=-\infty$, then for $p:=\mathbf{P}\left[g=-L_{g}\right]>0$ we have

$$
\mathbf{E}[u(w+g)]-u(w) \leq p u\left(w-L_{g}\right)+(1-p) u\left(w+M_{g}\right)-u(w) \rightarrow-\infty
$$

as $w$ decreases to $L_{g}$. Therefore $\mathbf{E}[u(w+g)]-u(w)<0$ for all $w$ close enough to $L_{g}$, and $g$ is rejected there.

If $u\left(0^{+}\right)>-\infty$ then let $0<\varepsilon<1 / 2$ be small enough so that $(1-\varepsilon) u(2)+$ $\varepsilon u\left(0^{+}\right)>u(1)$ (recall that $u$ is increasing). Then the gamble $g \in \mathcal{G}$ that takes the values 1 and -1 with probabilities $1-\varepsilon$ and $\varepsilon$, respectively, is accepted by $u$ at all $w>1=L_{g}$ that are close enough to 1 .

These two implications, together with the fact that the clauses in (i) and (ii) on rejection and acceptance, respectively, are clearly contradictory, yield the converse implications in both (i) and (ii). QED

Corollary 7. Let $u \in \mathcal{U}_{\mathrm{DA}}$, particularly $u \in \mathcal{U}^{*}$; then $u \in \mathcal{U}_{\mathrm{sr}}$ if and only if $u\left(0^{+}\right)=-\infty$.

Proof. In case (i) of proposition 6 we get some $d \equiv d_{g}>0$, possibly $d=\infty$, such that $u$ rejects $g$ at all $w \leq L_{g}+d$ and accepts $g$ at all $w>L_{g}+d$; in case (ii), $u$ accepts $g$ at all $w>L_{g}$ (and so $d_{g}=0$ ) - therefore $g$ is sometimes rejected if and only if case (i) occurs. QED

Lemma 8. Let $u \in \mathcal{U}$. If there is $c>0$ such that $\sup _{0<x \leq c} \tilde{\rho}_{u}(x)<1$ then $u\left(0^{+}\right)>-\infty$.

\footnotetext{
${ }^{38}$ The limit exists (and is either finite or $-\infty$ ) since $u$ is an increasing function.
} 
Proof. Let $\gamma:=\sup _{0<x \leq c} \tilde{\rho}_{u}(x)$; then $0 \leq \gamma<1$. For every $x \in(0, c]$ we have

$$
\left(\log u^{\prime}(x)\right)^{\prime}=-\rho_{u}(x)=-\tilde{\rho}_{u}(x) / x \geq-\gamma / x,
$$

and so

$$
\log u^{\prime}(c)-\log u^{\prime}(x) \geq \int_{x}^{c}(-\gamma / y) d y=-\gamma \log (c / x) .
$$

Therefore $u^{\prime}(x) \leq a x^{-\gamma}$ (with $a:=u^{\prime}(c) c^{\gamma}>0$ ), from which we get

$$
u(c)-u(x) \leq \int_{x}^{c} a y^{-\gamma} d y=a\left(c^{1-\gamma}-x^{1-\gamma}\right) /(1-\gamma),
$$

and so $u(x) \geq B:=u(c)-a c^{1-\gamma} /(1-\gamma)$ and $u\left(0^{+}\right) \geq B$. QED

Corollary 9. If $u \in \mathcal{U}^{*}$ then $\tilde{\rho}_{u}(x) \geq 1$ for all $x>0$.

Proof. If $\tilde{\rho}_{u}(c)<1$ for some $c>0$, then $\tilde{\rho}_{u}(x) \leq \gamma:=\tilde{\rho}_{u}(c)<1$ (since $u \in \mathcal{U}_{\mathrm{IR}}$ and so $\tilde{\rho}_{u}$ is increasing); lemma 8 implies that $u\left(0^{+}\right)$is finite, and so $u \notin \mathcal{U}_{\text {sr }}$ by corollary 7. QED

Lemma 10. Let $u \in \mathcal{U}^{*}$ and $g \in \mathcal{G}$. Then $u$ rejects $g$ at all $w \leq R^{\mathrm{FH}}(g)$.

Proof. By the definition of the Foster-Hart measure, $\tilde{v}_{1}(x)=\log (x)$ rejects $g$ at all $w \leq R^{\mathrm{FH}}(g)$. The result follows from proposition 2 with $u_{1}=u$ and $u_{2}=$ $\tilde{v}_{1}$ (and so $\tilde{\rho}_{2}(x)=1$ for every $x>0$ ), and $I=(0, \infty)$. QED

We can now prove theorem 1 (ii).

Proof of theorem 1(ii). Lemma 10, together with the fact that at each $w>$ $R^{\mathrm{FH}}(g)$ the CRRA utility $\tilde{v}_{1}=\log$, which belongs to $\mathcal{U}^{*}$, does not reject $g$, implies that " $g$ is rejected by all $u \in \mathcal{U}^{*}$ at $\mathrm{w}$ " if and only if " $w \leq R^{\mathrm{FH}}(g)$." So the condition in [UU] translates to "if $w \leq R^{\mathrm{FH}}(g)$ then $w \leq R^{\mathrm{FH}}(h)$," which is equivalent to " $R^{\mathrm{FH}}(g) \leq R^{\mathrm{FH}}(h)$." QED

Remarks. (1) The proofs above show that for the utility-uniform results one can replace $\mathcal{U}^{*}$ with any collection of utilities $\mathcal{U}^{* \mathrm{U}} \subset \mathcal{U}$ that satisfies: (i) $\tilde{v}_{1} \equiv$ $\log \in \mathcal{U}^{* \mathrm{U}}$ and (ii) $\inf _{x>0} \tilde{\rho}_{u}(x) \geq 1$ for every $u \in \mathcal{U}^{* \mathrm{U}}$. In particular, one may take $\mathcal{U}^{* \mathrm{U}}=\mathcal{U}_{\mathrm{IR}} \cap \mathcal{U}_{\mathrm{sr}}$, or $\mathcal{U}^{* \mathrm{U}}=\mathcal{U}_{\mathrm{CR}} \cap \mathcal{U}_{\mathrm{sr}^{\prime}}$, where $\mathcal{U}_{\mathrm{sr}^{\prime}}$ requires rejection at arbitrarily small wealth levels (i.e., $u \in \mathcal{U}_{\text {sr' }^{\prime}}$ if and only if for every $g \in \mathcal{G}$ and $\delta>0$ there is $w \in\left(L_{g}, L_{g}+\delta\right)$ such that $u$ rejects $g$ at $\left.w\right)$.

(2) If we drop the condition that each utility function $u$ will sometimes reject any gamble-i.e., $u \in \mathcal{U}_{\mathrm{sr}}$ - then the only wealth levels $w$ where all utilities reject a gamble $g$ are $w \leq L_{g}$, and so the resulting order, denote it $\geq_{L}$, satisfies $g \geq_{\mathrm{L}} h$ if and only if $L_{g} \leq L_{h}{ }^{39}$

\section{References}

Arrow, Kenneth J. 1965. Aspects of the Theory of Risk-Bearing. Helsinki: Yrjö Jahnssonin Säätiö.

. 1971. Essays in the Theory of Risk Bearing. Chicago: Markham.

$\rightarrow$ Aumann, Robert J., and Roberto Serrano. 2008. "An Economic Index of Riskiness." J.P.E. 116:810-36.

$\rightarrow$ Dybvig, Philip H., and Steven A. Lippman. 1983. "An Alternative Characterization of Decreasing Absolute Risk Aversion.” Econometrica 51:223-24.

${ }^{39}$ Interestingly, in the axiomatic approach of Foster and Hart (2011), replacing monotonicity with weak monotonicity yields $L$ instead of $R^{\mathrm{FH}}$. 
$\rightarrow$ Foster, Dean P., and Sergiu Hart. 2009. "An Operational Measure of Riskiness." J.P.E. 117:785-814.

_. 2011. "A Wealth-Requirement Axiomatization of Riskiness." Discussion Paper no. 577, Hebrew Univ. Jerusalem, Center Study Rationality, http:// www.ma.huji.ac.il/hart/abs/risk-ax.html.

$\rightarrow$ Hadar, Josef, and William Russell. 1969. "Rules for Ordering Uncertain Prospects." A.E.R. 59:25-34.

$\rightarrow$ Hanoch, Giora, and Haim Levy. 1969. "The Efficiency Analysis of Choices Involving Risk.” Rev. Econ. Studies 36:335-46.

Hart, Sergiu. 2009. "A Simple Riskiness Order Leading to the Aumann-Serrano Index of Riskiness.” Discussion Paper no. 517, Hebrew Univ. Jerusalem, Center Study Rationality, http://www.ma.huji.ac.il/hart/abs/risk-as.html.

- 2010. "Comparing Risks by Acceptance and Rejection." Discussion Paper no. 531, Hebrew Univ. Jerusalem, Center Study Rationality, http://www.ma .huji.ac.il/hart/abs/risk-u.html.

$\rightarrow$ Kahneman, Daniel, and Amos Tversky. 1979. "Prospect Theory: An Analysis of Decision under Risk." Econometrica 47:263-91.

$\rightarrow$ Levy, Haim. 1992. "Stochastic Dominance and Expected Utility: Survey and Analysis." Management Sci. 38:555-93.

Machina, Mark J., and Michael Rothschild. 2008. "Risk." In The New Palgrave Dictionary of Economics, 2nd ed., edited by S. N. Durlauf and L. E. Blume. London: Palgrave Macmillan.

$\rightarrow$ Meilijson, Isaac. 2009. "On the Adjustment Coefficient, Drawdowns and Lundberg-Type Bounds for Random Walk.” Ann. Appl. Probability 19:1015-25.

$\rightarrow$ Meyer, Donald J., and Jack Meyer. 2005. "Relative Risk Aversion: What Do We Know?" J. Risk and Uncertainty 31:243-62.

$\rightarrow \longrightarrow$. 2006. "Measuring Risk Aversion." Foundations and Trends in Microeconomics 2:107-203.

$\rightarrow$ Palacios-Huerta, Ignacio, and Roberto Serrano. 2006. "Rejecting Small Gambles under Expected Utility." Econ. Letters 91:250-59.

Palacios-Huerta, Ignacio, Roberto Serrano, and Oscar Volij. 2004. "Rejecting Small Gambles under Expected Utility." Manuscript, Brown Univ., Dept. Econ.

$\rightarrow$ Pratt, John W. 1964. "Risk Aversion in the Small and in the Large." Econometrica 32:122-36.

$\rightarrow$ Rabin, Matthew. 2000. "Risk Aversion and Expected-Utility Theory: A Calibration Theorem." Econometrica 68:1281-92.

$\rightarrow$ Rothschild, Michael, and Joseph Stiglitz. 1970. "Increasing Risk: I. A Definition." J. Econ. Theory 2:225-43.

$\rightarrow+$. 1971. "Increasing Risk: II. Its Economic Consequences." J. Econ. Theory 3:66-84.

$\rightarrow$ Whitmore, G. A. 1970. "Third-Degree Stochastic Dominance." A.E.R. 60: 45759.

$\rightarrow$ Yaari, Menahem E. 1969. "Some Remarks on Measures of Risk Aversion and on Their Uses.” J. Econ. Theory 1:315-29. 